\title{
Processing of THz images acquired by a passive camera
}

\author{
Marcin Kowalski, ${ }^{* 1}$ Norbert Palka, ${ }^{1}$ Marek Piszczek, ${ }^{1}$ Mieczyslaw Szustakowski ${ }^{1}$ \\ ${ }^{1}$ Institute of Optoelectronics, Military University of Technology, Kaliskiego 2, 00-908 Warszawa
}

Received August 15, 2012; accepted September 25, 2012; published September 30, 2012

\begin{abstract}
The terahertz (THz) band has lately become very interesting for scientists mainly because of its specific properties. One of the potential applications of $\mathrm{THz}$ devices is the security area. Imaging devices working in the $\mathrm{THz}$ range can be used to detect objects hidden under clothes. Since most of the commercially available $\mathrm{THz}$ cameras offer low quality images, a great possibility for digital processing techniques in the field of image quality improvement as well as detection of threats is expected. In the paper various image processing methods and algorithms of $\mathrm{THz}$ images are presented and analyzed.
\end{abstract}

$\mathrm{THz}$ electromagnetic waves offer great possibilities for security applications ranging from remote detection of chemicals (e.g. explosives) to visualization of objects hidden on persons under clothes. The detection of hidden items (like bombs, guns, drugs, etc.) is a very urgent and demanding problem in security and defense applications. This problem is very interesting from a hardware as well as a software point of view. The development of $\mathrm{THz}$ imaging technology is needed because of the low quality of commercially available cameras. The basic parameters of recent $\mathrm{THz}$ cameras such as the field of view or pixel resolution are lower than in the case of cameras in other spectral bands. However, an improvement of the $\mathrm{THz}$ image quality can be achieved using various image processing methods and algorithms.

For our studies, we chose a TS4 camera from ThruVision Systems Ltd. [1], which offers quite good quality images with a pixel resolution of $140 \times 240$ points. The camera operates at a frequency of $0.25 \mathrm{THz}$ and detects $\mathrm{THz}$ radiation thanks to the fact that clothing transmits well in this range. A heterodyne detection method is based on a GaAs Schottky mixer combined with a local oscillator. The operating distance of a camera-target is set to a range of 3-15 meters, however investigation shows that a useful detection distance is lower. TS4 can detect objects hidden under clothing (bombs, guns, knives, wallets, belts, etc.) due to their different temperature and emissivity than those of a human body. In the middle of the $\mathrm{THz}$ aperture, a small visible camera is installed to observe the scene.

Testing $\mathrm{THz}$ cameras usually requires some persons carrying hidden guns, bombs or knives. Such persons can have different clothes or body temperature, which makes the measurements even more ambiguous [2]. To avoid

*E-mail: mkowalski@wat.edu.pl such long-lasting and tiresome investigations we built a moving platform with a human phantom, which mimics a moving human being (Fig. 1).

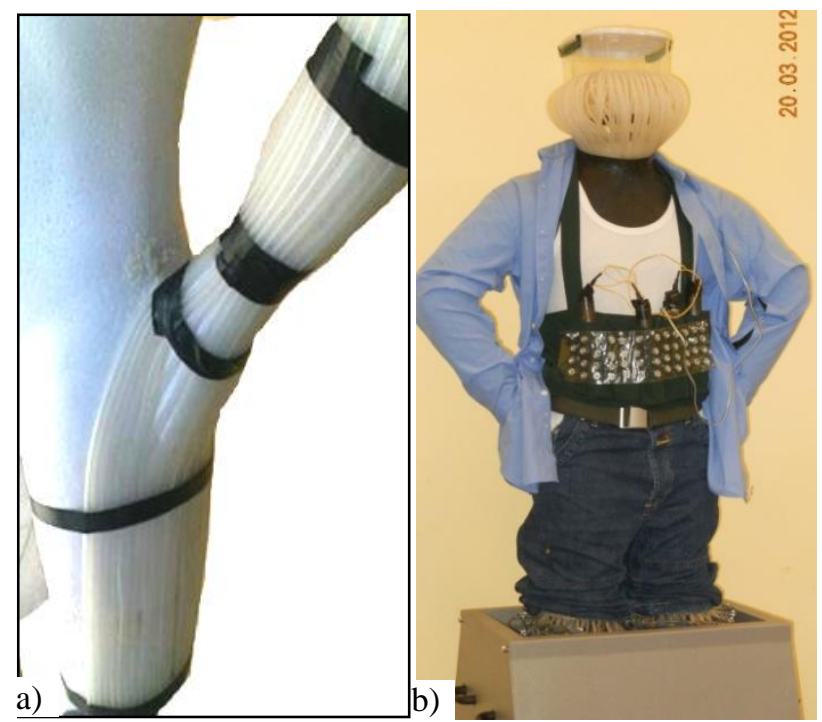

Fig. 1. The thermal phantom: partially covered with pipes (a) and readyto-use with a body-borne improvised explosive device (b).

There are at least two problems in processing and analysis of $\mathrm{THz}$ images. One is a selection of functions and parameters for the best fit between a model and its image data. The other is optimum scheduling of compute resources for the execution of the processing loops needed for fitting the model parameters to the image data [3]-[4].

Our goals are to detect objects hidden under clothes and improve quality of $\mathrm{THz}$ images. For these reasons various tests were prepared. At the beginning, we selected a set of images acquired by the $\mathrm{THz}$ camera. All these images show the phantom or a man (height: $188 \mathrm{~cm}$, weight: 95 $\mathrm{kg}$ ). The distance between the target and the camera was 4 meters. The sample images of the phantom and the man are shown in Fig. 2. One can notice that we obtained satisfactory similarities between both targets. The phantom image seems to be more homogeneous.

Figure 3 presents $\mathrm{THz}$ images taken for the phantom and the man wearing trousers and a cotton shirt plus a $30 \mathrm{~cm}$ long steel knife (Fig. 3 - an inset). The distance to 
the target was about 4 meters. It can be concluded that the similarities are satisfactory also in this case.
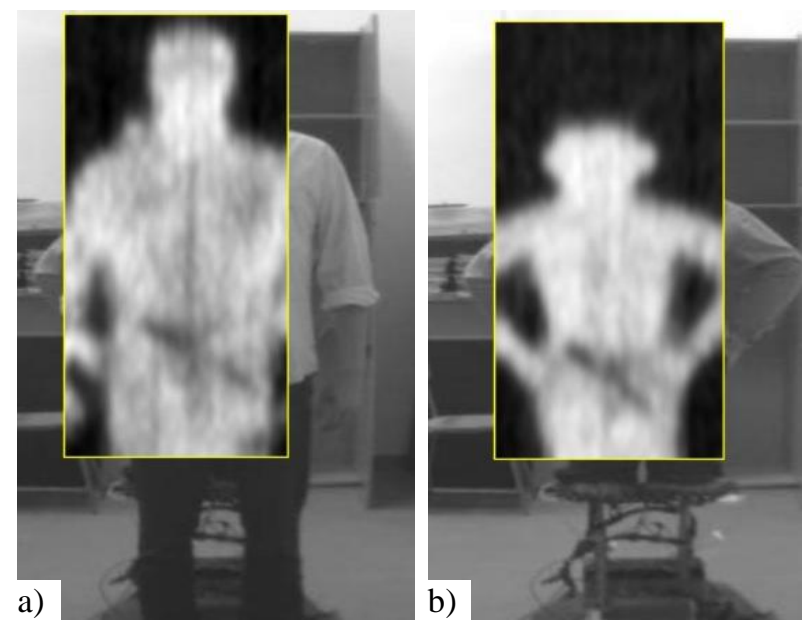

Fig. 2 Passive THz images taken with a THz camera for:a person (left) and a thermal phantom (right). One can notice a knife hidden under the cotton shirt.

Another object used as a possible threat was a metallic box hidden under the cotton shirt. Images were registered with a distance between the target and the camera of 4 meters. Moreover, we show the initial results of image processing, which can improve the contrast of images and determine the location of a hidden object in a more precise way. The images were analyzed by different techniques including image deblurring, various segmentation techniques, median filtration, and edge detection.

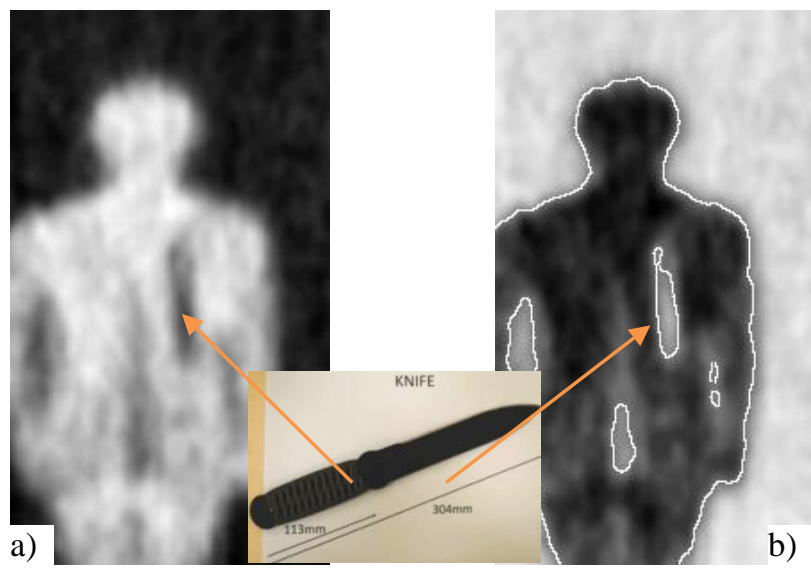

Fig. 3. THz images of a knife hidden under a shirt: original (b) and processed (b).

Figure 3 presents the results of THz image processing of the phantom with a hidden knife described above. Due to a low resolution of the $\mathrm{THz}$ camera, the shape of detected objects can differ from the original object shapes. Nevertheless, even small and thin objects can be easily detected by our algorithms. Images taken with the $\mathrm{THz}$ camera as well as processed images of the phantom with a $30 \mathrm{~cm}$ metallic knife are shown in Fig. 3. Figure 4 presents the original and processed $\mathrm{THz}$ images of the metallic box hidden under a shirt. It is clearly seen that the application of the developed algorithms can improve the contrast of images and determine the location of a hidden object in a more precise way.

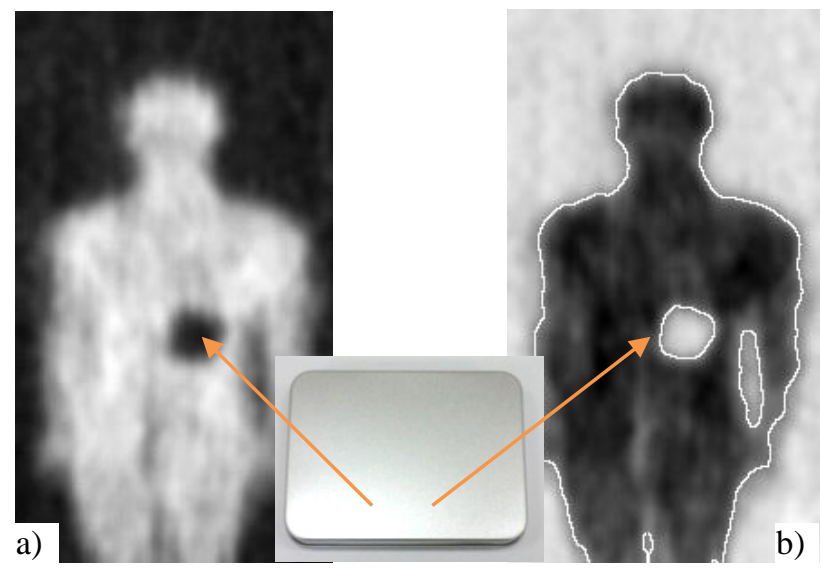

Fig. 4. THz Images of a mettalic box hidden under a shirt: original (b) and processed (b).

The presented images are the results of using various image processing methods and algorithms. The first step of processing of $\mathrm{THz}$ images is the application of various thresholding algorithms. These methods include both global and local thresholding algorithms with various thresholds. Next, the resultant binary images are processed using different edge detection algorithms. The last step during an image transformation process is image smoothing. There are many different algorithms that can be applied to improve the smoothness of an image. After comparison of many tests, taking into account hardware implementation issues, a filter based on a principal component analysis method was chosen and prepared [5].

The images presented in the paper show that there is a huge potential for $\mathrm{THz}$ image processing methods. One of the most important aspects of the presented results is that all of these images were processed with methods which can be efficiently implemented in a camera and work in a real-time mode. From many sets of methods the best and the most efficient algorithms were selected and finally implemented in the camera. According to laboratory test results images can be processed in real-time with a camera operating with 30 frames per second.

Considering the fact that even $\mathrm{THz}$ images with a low pixel resolution still provide valuable information, it is justified to combine them with high-resolution images from a visible camera. Image fusion allows us to combine the most interesting elements of images from various spectral ranges into one image [6]. Image processing 
methods and algorithms for the fusion of $\mathrm{THz}$ and visible (VIS) images are based on those presented above.

Since the $\mathrm{THz}$ and VIS images have different fields of view and sizes (140x240 pixels and 600x550 pixels, respectively) we need to find a common area of both images. This operation is very often connected with geometrical transformations of images.

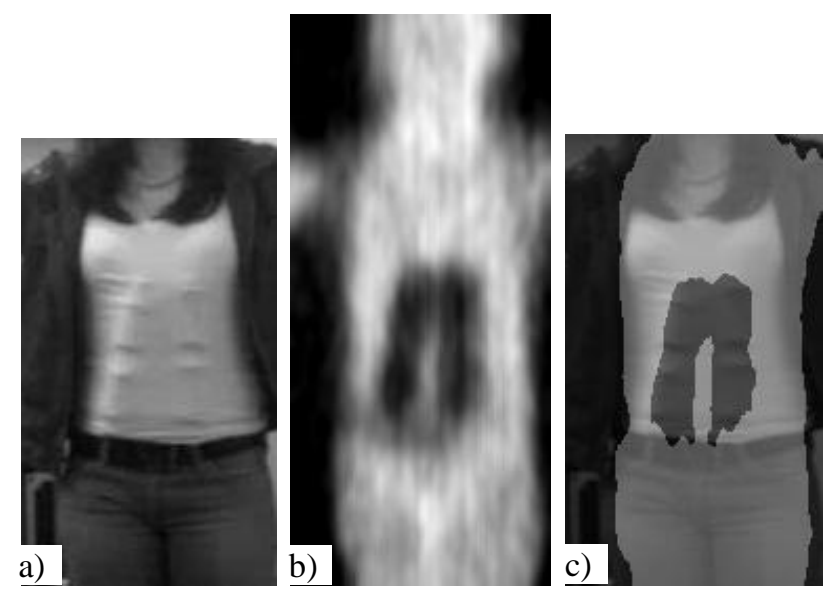

Fig. 5. Three stages of combining images of a person with hidden items: resized VIS image (a), THz image (b), combination of THz and VIS images (c).

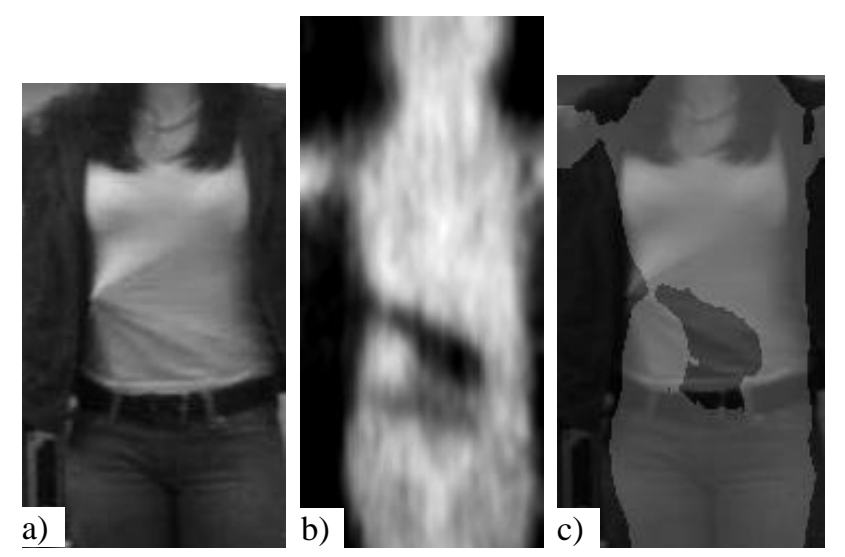

Fig. 6. Three stages of combining images with a hidden gun: resized VIS image (a), THz image (b), combination of THz and VIS images (c)

The images presented in Fig. 5 and Fig. 6 show the results of fusion of raw VIS and $\mathrm{THz}$ images. These images were combined using a Haar wavelet with averaging approximation [7]. One can notice that the resulted images show features of both the $\mathrm{THz}$ and VIS images, which was basically the goal of the investigations. Future research will focus on further development of $\mathrm{THz}$ and VIS fusion as well as combination of $\mathrm{THz}$, VIS and infrared images.
The results presented in this paper show that computer processing of images from a $\mathrm{THz}$ camera is a really promising and cost-effective way for demanding security and defense applications. The exploitation of exploratory data analysis tools and preparation of predictive models is the next step in the future research.

During the investigation process many image processing methods and algorithms were tested taking into account effectiveness and efficiency. The effectiveness of developed algorithms and code depends mainly on the distance between the human body and the camera, size of object and its type. It should be highlighted that the use of only one image processing method for all situations can be ineffective. To solve the problem of image quality enhancement we need to combine various algorithms.

A very promising part of our research is image fusion. Our recent investigations on combining $\mathrm{THz}$ and VIS images show that there is a great potential in combining two images from different spectral ranges, especially for security applications. In this part of our research we focus on extracting the most valuable features from each image and combing them into one. Future research will lead to further development of image fusion algorithms as well as combination of VIS, THz and infrared images.

It should be noted that all image processing methods and algorithms can be done in real-time with a camera operating 30 frames per second.

We acknowledge the financial support from the Polish Ministry of Science and Education under the Project $\mathrm{O}$ N515 020140.

\section{References}

[1] ThruVision System Ltd. website: http://www.thruvision.com.

[2] N. Palka et al., IEEE Microwave Radar and Wireless Communications (MIKON), 2012 19th International Conference on, 265 (2012).

[3] V.A. Trofimov et al., Proc. SPIE 8189, 81890I (2011).

[4] L. Yujiri, M. Shoucri, P. Moffa, IEEE Microwave Magazine 39, N5, 29 (2003).

[5] N.N. Wang, J.H. Qiu, W.B. Deng, Infrared Technology 31, N3, 129 (2009).

[6] M. Piszczek, M. Kowalski, M. Szustakowski, Acta Phys. Pol. A 120, 720 (2011).

[7] C. Toxqui-Quitl et al., Proc. SPIE 5558, 796 (2004). 